\title{
ON A CLASS OF PARTITIONS WITH DISTINCT SUMMANDS
}

\author{
BY
PETER HAGIS, JR.
}

\section{INTRODUCTION}

1. Let $p$ be an odd prime, and let $a=\left\{a_{1}, a_{2}, \cdots, a_{r}\right\}$, where $1 \leqq a_{j} \leqq(p-1) / 2$, be a set of $r$ distinct integers. In an earlier paper [1] the author has obtained a convergent series representation and asymptotic formulas for $p_{a}(n)$, the number of partitions of a positive integer $n$ into summands congruent to $\pm a_{j}(\bmod p)$. The necessary transformation equations as well as estimates of the magnitude of certain exponential sums were obtained using the procedures of Lehner [2], while the circle dissection method of Rademacher [3] was employed for the integration. In the present paper we impose an additional restriction on the partitions, namely that the summands be distinct. That is, we wish to find a convergent series and asymptotic formulas for $q_{a}(n)$, the number of partitions of a positive integer $n$ into distinct summands which are congruent modulo $p$ to elements of $a$ or their negatives. The methods employed are essentially the same as in [1] and free use will be made of the results obtained in the earlier paper whenever they are applicable.

2. In the sequel several generating functions, each convergent in the interior of the unit circle, will be needed. For convenience we list these now.

$$
\begin{aligned}
F_{a}(x) & =\prod_{j=1}^{r}\left(\prod_{m=0}^{\infty}\left(1-x^{p m+a_{j}}\right)^{-1} \prod_{m=1}^{\infty}\left(1-x^{p m-a_{j}}\right)^{-1}\right) \\
& =1+\sum_{n=1}^{\infty} p_{a}(n) x^{n} . \\
G_{a}(x) & =\prod_{j=1}^{r}\left(\prod_{m=0}^{\infty}\left(1+x^{p m+a_{j}}\right) \prod_{m=1}^{\infty}\left(1+x^{p m-a_{j}}\right)\right) \\
& =F_{a}(x) / F_{a}\left(x^{2}\right)=1+\sum_{n=1}^{\infty} q_{a}(n) x^{n} .
\end{aligned}
$$

Let $k$ be a positive integer and let $h$ satisfy the conditions $(h, k)=1,0 \leqq h<k$. If $p \mid k$ we define $b_{j} \equiv \pm h a_{j}(\bmod p)$, whichever yields $1 \leqq b_{j} \leqq(p-1) / 2$, and also define

$$
b=\left\{b_{1}, b_{2}, \cdots, b_{r}\right\} .
$$

Received by the editors April 22, 1963. 
Our third function is

$$
\begin{aligned}
I_{b}(x) & =\prod_{j=1}^{r}\left(\prod_{m=1}^{\infty}\left(1-x^{(2 m-1) p+2 b_{j}}\right)\left(1-x^{(2 m-1) p-2 b_{j}}\right)\right) \\
& =1+\sum_{n=1}^{\infty} s_{b}(n) x^{n} .
\end{aligned}
$$

If $p \nmid k$ we define $\alpha_{j}$ by

$$
\alpha_{j} k \equiv a_{j}(\bmod p), 0<\alpha_{j}<p
$$

and

$$
\rho_{j}=\exp \left(2 \pi i \alpha_{j} / p\right), \quad \bar{\rho}_{j}=\exp \left(-2 \pi i \alpha_{j} / p\right) .
$$

Then, we define

$$
\begin{aligned}
H_{a k}(x) & =\prod_{j=1}^{r}\left(\prod_{m=1}^{\infty}\left(1-\rho_{j} x^{m}\right)^{-1}\left(1-\bar{\rho}_{j} x^{m}\right)^{-1}\right) \\
& =1+\sum_{n=1}^{\infty} c_{a k}(n) x^{n}, \\
J_{a k}(x) & =\prod_{j=1}^{r}\left(\prod_{m=1}^{\infty}\left(1+\rho_{j} x^{m}\right)\left(1+\bar{\rho}_{j} x^{m}\right)\right) \\
& =1+\sum_{n=1}^{\infty} d_{a k}(n) x^{n}, \\
P_{a k}(x) & =\prod_{j=1}^{r}\left(\prod_{m=1}^{\infty}\left(1-\rho_{j} x^{2 m-1}\right)\left(1-\bar{\rho}_{j} x^{2 m-1}\right)\right) \\
& =1+\sum_{n=1}^{\infty} e_{a k}(n) x^{n} .
\end{aligned}
$$

\section{The Transformation Equations}

3. In what follows it will be necessary to determine the behavior of $G_{a}(x)$ in the neighborhood of a rational point on a circle concentric to the unit circle and of radius less than 1 . Therefore, we take

$$
x=\exp \{2 \pi i h / k-2 \pi z / k\}
$$

where $\mathscr{R}(z)>0,(h, k)=1,0 \leqq h<k$.

Theorem 1 of [1] states that

$$
\begin{aligned}
F_{a}(\exp \{2 \pi i h / k-2 \pi z / k\})= & \omega_{a}(h, k) \exp \{\pi(B / z-A z) / 6 p k\} \\
& \cdot F_{b}\left(\exp \left\{2 \pi i h^{\prime} / k-2 \pi / z k\right\}\right)
\end{aligned}
$$

if $p \mid k$, and 


$$
F_{a}(\exp \{2 \pi i h / k-2 \pi z / k\})=2^{-r} \chi_{a}(h, k) \prod_{j=1}^{r} \csc \pi \alpha_{j} / p
$$

if $p \nmid k$.

$$
\cdot \exp \{\pi(r / z-A z) / 6 p k\} H_{a k}\left(\exp \left\{2 \pi i H^{\prime} / k-2 \pi / z K\right\}\right)
$$

Here,

$$
\begin{aligned}
A & =\sum_{j=1}^{r}\left(p^{2}-6 a_{j} p+6 a_{j}^{2}\right) \\
B & =\sum_{j=1}^{r}\left(p^{2}-6 b_{j} p+6 b_{j}^{2}\right) \\
\omega_{a}(h, k) & =\exp \left\{\pi i \sigma_{a}(h, k)\right\} \\
\sigma_{a}(h, k) & =\sum_{j=1}^{r} \sum_{\mu_{j}}\left(\left(\mu_{j} / k\right)\right)\left(\left(h \mu_{j} / k\right)\right), 0<\mu_{j}<k, \mu_{j} \equiv \pm a_{j}(\bmod p) \\
\chi_{a}(h, k) & =\exp \left\{\pi i t_{a}(h, k)\right\}, \\
t_{a}(h, k) & =\sum_{j=1}^{r} \sum_{\mu_{j}}\left(\left(\mu_{j} / K\right)\right)\left(\left(h \mu_{j} / k\right)\right), 0<\mu_{j}<K, \mu_{j} \equiv \pm a_{j}(\bmod p) .
\end{aligned}
$$

$((y))=y-[y]-\frac{1}{2}+\frac{1}{2} \delta(y)$ where $\delta(y)=1$ if $y$ is an integer and 0 otherwise. $b$ and $\alpha_{j}$ are given by (2.3) and (2.5) respectively.

With the aid of this theorem we can now derive the necessary transformation equations for $G_{a}(x)$, the generating function of $q_{a}(n)$. Four cases must be considered. If $2 p \mid k$ and $x$ is given by (3.1) then $x^{2}=\exp \left\{2 \pi i h / k^{*}-2 \pi z / k^{*}\right\}$ where $2 k^{*}=k$. Since $G_{a}(x)=F_{a}(x) / F_{a}\left(x^{2}\right)$ we have, applying (3.2),

where

$$
G_{a}(x)=w_{a}(h, k) \exp \{\pi(A z-B / z) / 6 p k\} G_{b}\left(x^{\prime}\right),
$$

$$
x^{\prime}=\exp \left\{2 \pi i h^{\prime} / k-2 \pi / z k\right\} .
$$

$h^{\prime}$ is a fixed solution of

$$
h h^{\prime} \equiv-1(\bmod k)
$$

and

$$
w_{a}(h, k)=\omega_{a}(h, k) / \omega_{a}\left(h, k^{*}\right) .
$$

If $p \mid k$ and $k$ is odd then $x^{2}=\exp \{4 \pi i h / k-4 \pi z / k\}$. From (3.2) it follows that

$$
G_{a}(x)=W_{a}(h, k) z \exp \{\pi(A z+B / z-D / 2 z) / 6 p k\} F_{b}\left(x^{\prime}\right) / F_{d}\left(y^{\prime}\right)
$$

where

$$
y^{\prime}=\exp \left\{2 \pi i g^{\prime} / k-\pi / z k\right\},
$$

$g^{\prime}$ is a fixed solution of 


$$
2 h g^{\prime} \equiv-1(\bmod k) \text {, }
$$

and

$$
W_{a}(h, k)=\omega_{a}(h, k) / \omega_{a}(2 h, k) .
$$

$d=\left\{d_{1}, d_{2}, \cdots, d_{r}\right\}$ where $d_{j} \equiv \pm 2 h a_{j}(\bmod p)$, whichever yields $1 \leqq d_{j} \leqq(p-1) / 2$, and $D=\sum_{j=1}^{r}\left(p^{2}-6 d_{j} p+6 d_{j}^{2}\right)$. Since $b_{j} \equiv \pm h a_{j}(\bmod p)$ it follows that $d_{j}=2 b_{j}$ or $d_{j}=p-2 b_{j}$. Thus, $D=\sum_{j=1}^{r}\left(p^{2}-12 b_{j} p+24 b_{j}^{2}\right)$. From (3.11) and (3.14) we have $h^{\prime} \equiv 2 g^{\prime}(\bmod k)$ so that $x^{\prime}=y^{\prime 2}$. We now easily verify that $F_{b}\left(x^{\prime}\right) / F_{d}\left(y^{\prime}\right)=I_{b}\left(y^{\prime}\right)$ where $I_{b}(x)$ is given by (2.4). We conclude that

where

$$
G_{a}(x)=W_{a}(h, k) \exp \left\{\pi\left(A z+B^{*} / z\right) / 6 p k\right\} I_{b}\left(y^{\prime}\right)
$$

$$
B^{*}=\sum_{j=1}^{r}\left(p^{2} / 2-6 b_{j}^{2}\right) .
$$

If $k$ is even and $p \nmid k$ then from (3.3)

$$
\begin{aligned}
& G_{a}(x)= z_{a}(h, k) \prod_{j=1}^{r} \csc \pi \alpha_{j} / p \sin \pi \beta_{j} / p \exp \{\pi(A z-r / z) / 6 p k\} \\
& \cdot H_{a k}(X) / H_{a k^{*}}\left(X^{2}\right)
\end{aligned}
$$

where

$$
X=\exp \left\{2 \pi i H^{\prime} / k-2 \pi / z K\right\}
$$

Here,

$$
\begin{aligned}
H H^{\prime} & \equiv-1(\bmod k), H=p h, K=p k ; \\
z_{a}(h, k) & =\chi_{a}(h, k) / \chi_{a}\left(h, k^{*}\right) ;
\end{aligned}
$$

and $\beta_{j} k^{*} \equiv a_{j}(\bmod p), 0<\beta_{j}<p$.

Since $\alpha_{j} k \equiv a_{j}(\bmod p)$ and $2 k^{*}=k$ it follows that $2 \alpha_{j} \equiv \beta_{j}(\bmod p)$. From (2.6) and (2.7) we now see that $H_{a k^{*}}\left(X^{2}\right)=\prod_{j=1}^{r}\left(\prod_{m=1}^{\infty}\left(1-\rho_{j}^{2} X^{2 m}\right)^{-1}\left(1-\bar{\rho}_{j}^{2} X^{2 m}\right)^{-1}\right)$, from which we easily deduce that $H_{a k}(X) / H_{a k^{*}}\left(X^{2}\right)=J_{a k}(X)$ where $J_{a k}(x)$ is given by (2.8). We conclude that

$$
G_{a}(x)=(-1)^{\mu} 2^{r} z_{a}(h, k) \prod_{j=1}^{r} \cos \pi \alpha_{j} / p \exp \{\pi(A z-r / z) / 6 p k\} J_{a k}(X)
$$

where $\mu$ is the number of $\alpha_{j}$ such that $\alpha_{j}>p / 2$.

If $k$ is odd and $p \nmid k$ then from (3.3)

$$
G_{a}(x)=Z_{a}(h, k) \exp \{\pi(A z+r / 2 z) / 6 p k\} H_{a k}(X) / H_{a k}(Y)
$$

where 


$$
Y=\exp \left\{2 \pi i G^{\prime} / k-\pi / z K\right\}
$$

Here

$$
2 H G^{\prime} \equiv-1(\bmod k)
$$

and

$$
Z_{a}(h, k)=\chi_{a}(h, k) / \chi_{a}(2 h, k)
$$

From (3.18) and (3.21) we have $2 G^{\prime} \equiv H^{\prime}(\bmod k)$ so that $X=Y^{2}$. We now easily verify that $H_{a k}(X) / H_{a k}(Y)=P_{a k}(Y)$ where $P_{a k}(x)$ is given by (2.9). We conclude that

$$
G_{a}(x)=Z_{a}(h, k) \exp \{\pi(A z+r / 2 z) / 6 p k\} P_{a k}(Y) .
$$

With $x, x^{\prime}, y^{\prime}, X, Y$ given by (3.1), (3.10), (3.13), (3.17), (3.20) respectively we combine these results to obtain

THEOREM 1. $G_{a}(x)$ satisfies the transformation equations

$$
G_{a}(x)=w_{a}(h, k) \exp \{\pi(A z-B / z) / 6 p k\} G_{b}\left(x^{\prime}\right)
$$

if $p \mid k$ and $2 \mid k$,

$$
G_{a}(x)=W_{a}(h, k) \exp \left\{\pi\left(A z+B^{*} / z\right) / 6 p k\right\} I_{b}\left(y^{\prime}\right)
$$

if $p \mid k$ and $2 \times k$,

$$
G_{a}(x)=(-1)^{\mu} 2^{r} z_{a}(h, k) \prod_{j=1}^{r} \cos \pi \alpha_{j} / p \exp \{\pi(A z-r / z) / 6 p k\} J_{a k}(X)
$$

if $p \nmid k$ and $2 \mid k$, and

$$
G_{a}(x)=Z_{a}(h, k) \exp \{\pi(A z+r / 2 z) / 6 p k\} P_{a k}(Y)
$$

if $p \nmid k$ and $2 \nmid k$.

\section{Estimates of Four Exponential SUMS}

4. In what follows it will be necessary to have information concerning the magnitude of certain exponential sums involving $w_{a}(h, k), W_{a}(h, k), z_{a}(h, k)$ and $Z_{a}(h, k)$. Since the trivial estimate $O(k)$ will not suffice we now undertake an investigation of these sums. The method used is essentially that of Lehner, and for further details the interested reader is referred to [1] and [2]. We first consider the case $p \mid k$.

With $\sigma_{q}(h, k)$ given by (3.7) we have by $(5.3),(5.4),(5.5)$ of $[1]$ 


$$
\begin{aligned}
& 6 p k \sigma_{a}(h, k) \equiv 0(\bmod 3) \text { if } 3 \times k \\
& 6 p k \sigma_{a}(h, k) \equiv \sum_{j=1}^{r}\left(2 p+2 a_{j}+2 c_{j}-3\right)(\bmod 4) \text { if } 2 \nmid k \\
& 6 h k p \sigma_{a}(h, k)= h^{2}\left(A+\sum_{j=1}^{r}\left\{2 k^{2}+3 k\left(2 a_{j}-p\right)\right\}\right)+\left(B-r k^{2}\right) \\
&-3 h k \sum_{j=1}^{r}\left(2 c_{j}-p\right)-12 k p I .
\end{aligned}
$$

Here $I$ is an integer, and $c_{j}=b_{j}$ if $b_{j} \equiv h a_{j}(\bmod p), c_{j}=p-b_{j}$ if $b_{j}$ $\equiv-h a_{j}(\bmod p)$.

Now let $12 p=f G$ where $f$ is the greatest divisor of $12 p$ prime to $k$. If $k$ is even we have $f=3, G=4 p$ if $3 \nmid k$, and $f=1, G=12 p$ if $3 \mid k$. If $k$ is odd we have $f=12, G=p$ if $3 \times k$, and $f=4, G=3 p$ if $3 \mid k$. Taking $h^{\prime}$ so that $h h^{\prime} \equiv-1$ $(\bmod G k)$ we have after multiplying $(4.3)$ by $-h^{\prime}$

$$
6 p k \sigma_{a}(h, k) \equiv h s-h^{\prime} t-3 k \sum_{j=1}^{r}\left(2 c_{j}-p\right)(\bmod G k)
$$

where

$$
\begin{aligned}
& s=A+\sum_{j=1}^{r}\left\{2 k^{2}+3 k\left(2 a_{j}-p\right)\right\}, \\
& t=B-r k^{2} .
\end{aligned}
$$

From (4.1) and (4.2) we have

$$
6 p k \sigma_{a}(h, k) \equiv \sum_{j=1}^{r}\left(6 p+6 a_{j}+6 c_{j}-3\right)(\bmod f) .
$$

If $k$ is even we have $h h^{\prime} \equiv-1\left(\bmod G k^{*}\right)$, and since (4.1), (4.2), (4.3) hold with $k$ replaced by $k^{*}$ we have

$$
6 p k^{*} \sigma_{a}\left(h, k^{*}\right) \equiv h S-h^{\prime} T-3 k^{*} \sum_{j=1}^{r}\left(2 c_{j}-p\right)\left(\bmod G k^{*}\right)
$$

where

$$
\begin{aligned}
& S=A+\sum_{j=1}^{r}\left\{2 k^{* 2}+3 k^{*}\left(2 a_{j}-p\right)\right\} \\
& T=B-r k^{* 2}
\end{aligned}
$$

Since, when $k$ is even, $f=1$ or $f=3$ we have from (4.6) and the corresponding statement for $k^{*}$

$$
6 p k \sigma_{a}(h, k) \equiv 6 p k^{*} \sigma_{a}\left(h, k^{*}\right) \equiv 0(\bmod f)
$$


If we define $\phi$ by $f \phi \equiv 1(\bmod G k)$ we have also $f \phi \equiv 1\left(\bmod G k^{*}\right)$. It follows then from (4.4), (4.7), and (4.9) that

$$
6 p k \sigma_{a}(h, k) \equiv f \phi\left(s h-t h^{\prime}-6 k C^{*}+3 r p k\right)(\bmod 12 p k)
$$

and

$$
6 p k^{*} \sigma_{a}\left(h, k^{*}\right) \equiv f \phi\left(S h-T h^{\prime}-6 k^{*} C^{*}+3 r p k^{*}\right)\left(\bmod 12 p k^{*}\right)
$$

where $C^{*}=\sum_{j=1}^{r} c_{j}$. From (3.6)

$$
\begin{aligned}
\omega_{a}(h, k) & =\exp \left\{2 \pi i \cdot 6 p k \sigma_{a}(h, k) / 12 p k\right\} \\
& =\exp \left\{2 \pi i\left(3 \phi\left(r p-2 C^{*}\right) / G+\phi\left(s h-t h^{\prime}\right) / G k\right)\right\} \\
\omega_{a}\left(h, k^{*}\right) & =\exp \left\{2 \pi i\left(3 \phi\left(r p-2 C^{*}\right) / G+\phi\left(S h-T h^{\prime}\right) / G k^{*}\right)\right\} .
\end{aligned}
$$

From (3.12), (4.5), (4.8) it follows that

$$
w_{a}(h, k)=\exp \left\{2 \pi i\left(\phi\left(u h+v h^{\prime}\right) / G k\right)\right\}
$$

where

$$
\begin{aligned}
& u=r k^{2}-A, \\
& v=B+2 r k^{* 2} .
\end{aligned}
$$

5. With $h$ replaced by $2 h$ we have from (4.1), (4.2), (4.3) if $k$ is odd

$$
\begin{aligned}
6 p k \sigma_{a}(2 h, k) \equiv & 0(\bmod 3) \text { if } 3 \nmid k \\
6 p k \sigma_{a}(2 h, k) \equiv & \sum_{j=1}^{r}\left(2 p+2 a_{j}+2 e_{j}-3\right)(\bmod 4), \\
12 h k p \sigma_{a}(2 h, k)= & 4 h^{2}\left(A+\sum_{j=1}^{r}\left\{2 k^{2}+3 k\left(2 a_{j}-p\right)\right\}\right)+\left(D-r k^{2}\right) \\
& -6 h k \sum_{j=1}^{r}\left(2 e_{j}-p\right)-12 k p I .
\end{aligned}
$$

Here $e_{j}=d_{j}$ if $d_{j} \equiv 2 h a_{j}(\bmod p), e_{j}=p-d_{j}$ if $d_{j} \equiv-2 h a_{j}(\bmod p) . d_{j}$ and $D$ are defined in $\$ 3$.

If we now take $g^{\prime}$ so that $2 h g^{\prime} \equiv-1(\bmod G k)$ we have after multiplying (5.3) by $-g^{\prime}$

$$
6 p k \sigma_{a}(2 h, k) \equiv 2 h s-g^{\prime} Q-3 k \sum_{j=1}^{r}\left(2 e_{j}-p\right)(\bmod G k),
$$

where $s$ is given by (4.5) and

$$
Q=D-r k^{2}
$$


From (5.1) and (5.2) we have

$$
6 p k \sigma_{a}(2 h, k) \equiv \sum_{j=1}^{r}\left(6 p+6 a_{j}+6 e_{j}-3\right)(\bmod f) .
$$

Defining $\phi$ as before and $\Gamma$ by $G k \Gamma \equiv 1(\bmod f)$ we have from (4.4), (4.6), (5.4), (5.6) if $k$ is odd

$$
\begin{aligned}
6 p k \sigma_{a}(h, k) \equiv & f \phi\left(s h-t h^{\prime}-6 k C^{*}+3 r p k\right) \\
& +G k \Gamma\left(6 A^{*}+6 C^{*}+6 r p-3 r\right)(\bmod 12 p k)
\end{aligned}
$$

and

$$
\begin{aligned}
6 p k \sigma_{a}(2 h, k) \equiv & f \phi\left(2 s h-Q g^{\prime}-6 k E^{*}+3 r p k\right) \\
& +G k \Gamma\left(6 A^{*}+6 E^{*}+6 r p-3 r\right)(\bmod 12 p k)
\end{aligned}
$$

where $A^{*}=\sum_{j=1}^{r} a_{j}, E^{*}=\sum_{j=1}^{r} e_{j}$. From (3.6)

$$
\begin{aligned}
\omega_{a}(h, k)= & \exp \left\{2 \pi i \left(\Gamma\left(6 A^{*}+6 C^{*}+6 r p-3 r\right) / f\right.\right. \\
& \left.\left.-3 \phi\left(2 C^{*}-r p\right) / G+\phi\left(s h-t h^{\prime}\right) / G k\right)\right\}, \\
\omega_{a}(2 h, k)= & \exp \left\{2 \pi i \left(\Gamma\left(6 A^{*}+6 E^{*}+6 r p-3 r\right) / f\right.\right. \\
& \left.\left.-3 \phi\left(2 E^{*}-r p\right) / G+\phi\left(2 s h-Q g^{\prime}\right) / G k\right)\right\} .
\end{aligned}
$$

Since $h h^{\prime} \equiv-1(\bmod G k)$ and $2 h g^{\prime} \equiv-1(\bmod G k)$ we have $h^{\prime} \equiv 2 g^{\prime}(\bmod G k)$. It follows from (3.15), (4.5), and (5.5) that

(5.7) $W_{a}(h, k)=\exp \left\{2 \pi i\left(\Gamma\left(6 C^{*}-6 E^{*}\right) / f-3 \phi\left(2 C^{*}-2 E^{*}\right) / G+\phi\left(u^{\prime} h+v^{\prime} g^{\prime}\right) / G k\right)\right\}$, where

$$
\begin{aligned}
& u^{\prime}=-A-2 r k^{2}-6 k A^{*}+3 r p k, \\
& v^{\prime}=r k^{2}-2 B^{*},
\end{aligned}
$$

and $B^{*}$ is given by (3.16).

6. Turning to the case $p \nmid k$ we have by (6.2), (6.3), (6.4), (6.5) of [1], with $t_{a}(h, k)$ given by (3.9)

$$
\begin{aligned}
& 6 p k t_{a}(h, k) \equiv 0(\bmod 3) \text { if } 3 \ngtr k \\
& 6 p k t_{a}(h, k) \equiv \sum_{j=1}^{r}\left(p-p k+2 a_{j}+2 \alpha_{j}\right)(\bmod 4) \text { if } 2 \Varangle k, \\
& 6 p k t_{a}(h, k) \equiv 0(\bmod p), \\
& 6 h k p t_{a}(h, k)=h^{2}\left(A+\sum_{j=1}^{r}\left\{2 K^{2}+3 K\left(2 a_{j}-p\right)\right\}\right) \\
&+r\left(1-k^{2}\right)+6 h k \sum_{j=1}^{r} \alpha_{j}-12 k I,
\end{aligned}
$$


where $I$ is an integer. If $p=3$ then $(\bmod p)$ in (6.3) may be replaced by $(\bmod 9)$.

Now let $12 p=F g$ where $F$ is the greatest divisor of $12 p$ prime to $k$. If $k$ is even we have $F=3 p, g=4$ if $3 \nmid k$, and $F=p, g=12$ if $3 \mid k$. If $k$ is odd we have $F=12 p, g=1$ if $3 \nmid k$, and $F=4 p, g=3$ if $3 \mid k$. Taking $h^{\prime}$ so that $h h^{\prime} \equiv-1$ (mod $g k$ ) we have after multiplying (6.4) by $-h^{\prime}$

$$
6 p k t_{a}(h, k) \equiv h s-h^{\prime} t+6 k \alpha^{*}(\bmod g k)
$$

where

$$
\begin{aligned}
& s=A+\sum_{j=1}^{r}\left\{2 K^{2}+3 K\left(2 a_{j}-p\right)\right\}, \\
& t=r\left(1-k^{2}\right),
\end{aligned}
$$

and $\alpha^{*}=\sum_{j=1}^{r} \alpha_{j}$. From (6.1), (6.2), (6.3) we have

$$
6 p k t_{a}(h, k) \equiv 9 p^{2} \sum_{j=1}^{r}\left(2 a_{j}+2 \alpha_{j}+p-p k\right)(\bmod F) .
$$

If $k$ is even then $h h^{\prime} \equiv-1\left(\bmod g k^{*}\right)$ and since (6.1), (6.2), (6.3), (6.4) hold with $k$ replaced by $k^{*}$ and $\alpha_{j}$ replaced by $\beta_{j}$, where $\beta_{j}$ is defined in $\S 3$, we have

$$
6 p k^{*} t_{a}\left(h, k^{*}\right) \equiv h S-h^{\prime} T+6 k^{*} \beta^{*}\left(\bmod g k^{*}\right)
$$

where

$$
\begin{aligned}
& S=A+\sum_{j=1}^{r}\left\{2 K^{* 2}+3 K^{*}\left(2 a_{j}-p\right)\right\}, \\
& T=r\left(1-k^{* 2}\right) .
\end{aligned}
$$

Here $K^{*}=p k^{*}$ and $\beta^{*}=\sum_{j=1}^{r} \beta_{j}$.

Since, when $k$ is even, $F=p$ or $F=3 p$ we have from (6.7) and the corresponding statement for $k^{*}$

$$
6 p k t_{a}(h, k) \equiv 6 p k^{*} t_{a}\left(h, k^{*}\right) \equiv 0(\bmod F) .
$$

If we define $\Phi$ by $F \Phi \equiv 1(\bmod g k)$ then also $F \Phi \equiv 1\left(\bmod g k^{*}\right)$. It follows then from (6.5), (6.8), (6.10) that

$$
\begin{aligned}
6 p k t_{a}(h, k) & \equiv F \Phi\left(s h-t h^{\prime}+6 k \alpha^{*}\right)(\bmod 12 p k), \\
6 p k^{*} t_{a}\left(h, k^{*}\right) & \equiv F \Phi\left(S h-T h^{\prime}+6 k^{*} \beta^{*}\right)\left(\bmod 12 p k^{*}\right) .
\end{aligned}
$$

Then, from (3.8)

$$
\begin{aligned}
\chi_{a}(h, k) & =\exp \left\{2 \pi i\left(6 \Phi \alpha^{*} / g+\Phi\left(s h-t h^{\prime}\right) / g k\right)\right\} \\
\chi_{a}\left(h, k^{*}\right) & =\exp \left\{2 \pi i\left(6 \Phi \beta^{*} / g+\Phi\left(S h-T h^{\prime}\right) / g k^{*}\right)\right\}
\end{aligned}
$$


From (3.19), (6.6), (6.9) it then follows that

$$
z_{a}(h, k)=\exp \left\{2 \pi i\left(6 \Phi\left(\alpha^{*}-\beta^{*}\right) / g+\Phi\left(U h+V h^{\prime}\right) / g k\right)\right\},
$$

where

$$
\begin{aligned}
& U=r K^{2}-A, \\
& V=r+2 r k^{* 2} .
\end{aligned}
$$

7. With $h$ replaced by $2 h$ we have from $(6.1),(6.2),(6.3),(6.4)$ if $k$ is odd

$$
\begin{aligned}
& 6 p k t_{a}(2 h, k) \equiv 0(\bmod 3) \text { if } 3 \nmid k, \\
& 6 p k t_{a}(2 h, k) \equiv \sum_{j=1}^{r}\left(p-p k+2 a_{j}+2 \alpha_{j}\right)(\bmod 4), \\
& 6 p k t_{a}(2 h, k) \equiv 0(\bmod p), \\
& 12 h k p t_{a}(2 h, k) \equiv 4 h^{2}\left(A+\sum_{j=1}^{r}\left\{2 K^{2}+3 K\left(2 a_{j}-p\right)\right\}\right)+r\left(1-k^{2}\right) \\
&+12 h k \alpha^{*}-12 k I .
\end{aligned}
$$

If $p=3$ then $(\bmod p)$ in $(7.3)$ is replaced by $(\bmod 9)$.

If we now take $g^{\prime}$ so that $2 h g^{\prime} \equiv-1(\bmod g k)$ we have after multiplying (7.4) by $-g^{\prime}$

$$
6 p k t_{a}(2 h, k) \equiv 2 h s-g^{\prime} t+6 k \alpha^{*}(\bmod g k),
$$

where $s$ and $t$ are given by (6.6). From (7.1), (7.2), (7.3)

$$
6 p k t_{a}(2 h, k) \equiv 9 p^{2} \sum_{j=1}^{r}\left(2 a_{j}+2 \alpha_{j}+p-p k\right)(\bmod F) .
$$

Defining $\Phi$ as before and $\gamma$ by $g k \gamma \equiv 1(\bmod F)$ we have from $(6.5),(6.7),(7.5)$, (7.6) if $k$ is odd

$$
\begin{aligned}
& 6 p k t_{a}(h, k) \equiv F \Phi\left(s h-t h^{\prime}+6 k \alpha^{*}\right)+9 g k \gamma p^{2}\left(2 A^{*}+2 \alpha^{*}+r p-r p k\right)(\bmod 12 p k), \\
& 6 p k t_{a}(2 h, k) \equiv F \Phi\left(2 s h-t g^{\prime}+6 k \alpha^{*}\right)+9 g k \gamma p^{2}\left(2 A^{*}+2 \alpha^{*}+r p-r p k\right)(\bmod 12 p k) .
\end{aligned}
$$

From (3.8)

$$
\begin{aligned}
& \chi_{a}(h, k)=\exp \left\{2 \pi i\left(9 p^{2} \gamma\left(2 A^{*}+2 \alpha^{*}+r p-r p k\right) / F+6 \Phi \alpha^{*} / g+\Phi\left(s h-t h^{\prime}\right) / g k\right)\right\}, \\
& \chi_{a}(2 h, k)=\exp \left\{2 \pi i\left(9 p^{2} \gamma\left(2 A^{*}+2 \alpha^{*}+r p-r p k\right) / F+6 \Phi \alpha^{*} / g+\Phi\left(2 s h-t g^{\prime}\right) / g k\right)\right\} .
\end{aligned}
$$

Since $h^{\prime} \equiv 2 g^{\prime}(\bmod g k)$ we have from $(3.22),(6.6)$ and the fact that $g k \mid 2 K^{2}$,

$$
Z_{a}(h, k)=\exp \left\{2 \pi i \Phi\left(U^{\prime} h+V^{\prime} g^{\prime}\right) / g k\right\}
$$

where 


$$
\begin{aligned}
& U^{\prime}=-A-6 K A^{*}+3 r p K, \\
& V^{\prime}=r k^{2}-r .
\end{aligned}
$$

8. THEOREM 2. The sum

$$
S_{1}=\sum_{h \bmod k}^{\prime} w_{a}(h, k) \exp \left\{-2 \pi i\left(h n-h^{\prime} v\right) / k\right\},
$$

where $h \equiv \zeta(\bmod p), p \nmid \zeta ; \sigma_{1} \leqq h^{\prime}<\sigma_{2}(\bmod k), 0 \leqq \sigma_{1}<\sigma_{2} \leqq k ; 2 p \mid k$, is subject to the estimate $O\left(n^{1 / 3} k^{2 / 3+\varepsilon}\right)$ uniformly in $v, \zeta, \sigma_{1}, \sigma_{2}, a$.

THEOREM 3. The sum

$$
S_{2}=\sum_{h \bmod k}^{\prime} W_{a}(h, k) \exp \left\{-2 \pi i\left(h n-g^{\prime} v\right) / k\right\},
$$

where $h \equiv \zeta(\bmod p), p \nmid \zeta ; \sigma_{1} \leqq h^{\prime}<\sigma_{2}(\bmod k), 0 \leqq \sigma_{1}<\sigma_{2} \leqq k ; p \mid k, 2 \nmid k$, is subject to the estimate $O\left(n^{1 / 3} k^{2 / 3+\varepsilon}\right)$ uniformly in $v, \zeta, \sigma_{1}, \sigma_{2}, a$.

THEOREM 4. The sum

$$
S_{3}=\sum_{h \bmod k}^{\prime} z_{a}(h, k) \exp \left\{-2 \pi i\left(h n-H^{\prime} v\right) / k\right\},
$$

where $\sigma_{1} \leqq h^{\prime}<\sigma_{2}(\bmod k), 0 \leqq \sigma_{1}<\sigma_{2} \leqq k ; p \nmid k, 2 \mid k$, is subject to the estimate $O\left(n^{1 / 3} k^{2 / 3+\varepsilon}\right)$ uniformly in $v, \sigma_{1}, \sigma_{2}, a$.

THEOREM 5. The sum

$$
S_{4}=\sum_{h \bmod h}^{\prime} Z_{a}(h, k) \exp \left\{-2 \pi i\left(h n-G^{\prime} v\right) / k\right\},
$$

where $\sigma_{1} \leqq h^{\prime}<\sigma_{2}(\bmod k), 0 \leqq \sigma_{1}<\sigma_{2} \leqq k ; p \nmid k, 2 \nmid k$, is subject to the estimate $O\left(n^{1 / 3} k^{2 / 3+\varepsilon}\right)$ uniformly in $v, \sigma_{1}, \sigma_{2}, a$.

Here $\sigma_{1}, \sigma_{2}$ are integers and $\Sigma^{\prime}$ indicates that $h$ runs over integers prime to the modulus of the sum. $h^{\prime}, g^{\prime}, H^{\prime}, G^{\prime}$ are given by (3.11), (3.14), (3.18), (3.21) respectively.

Proof. $w_{a}(h, k), W_{a}(h, k), z_{a}(h, k), Z_{a}(h, k)$, when viewed as functions of $h$, all have period $k$. Therefore, if in $S_{1}$ and $S_{2}$ we select $h^{\prime}$ so that $h h^{\prime} \equiv-1(\bmod G k)$ and make use of (4.10) and (5.7) we can write

$$
\begin{aligned}
& S_{1}=G^{-1} \sum_{h \bmod G k}^{\prime} \exp \{\dot{2} \pi i f(h) / G k\}, \\
& S_{2}=G^{-1} c_{2}(a, k, \zeta) \sum_{h \bmod G k} \exp \{2 \pi i g(h) / G k\},
\end{aligned}
$$

where $\left|c_{2}\right|=1, \quad f(h)=(\phi u-G n) h+(\phi v+G v) h^{\prime}$, and $g(h)=\left(\phi u^{\prime}-G n\right) h$ $+\left(\phi v^{\prime}+G v\right) g^{\prime}$. Since $h^{\prime} \equiv 2 g^{\prime}(\bmod G k)$ and since an integer $\lambda$ exists such that $2 \lambda \equiv 1(\bmod G k)$ we see that $g(h) \equiv\left(\phi u^{\prime}-G n\right) h+\lambda\left(\phi v^{\prime}+G v\right) h^{\prime}(\bmod G k)$.

If in $S_{3}$ and $S_{4}$ we take $h^{\prime}$ so that $h h^{\prime} \equiv-1(\bmod g k)$ and make use of (6.11), (7.7) we can write 


$$
\begin{aligned}
& S_{3}=g^{-1} c_{3}(a, k) \sum_{h \bmod g k}^{\sum^{\prime}} \exp \{2 \pi i F(h) / g k\}, \\
& S_{4}=g^{-1} \sum_{h \bmod g k}^{\sum^{\prime}} \exp \{2 \pi i G(h) / g k\},
\end{aligned}
$$

where $\left|c_{3}\right|=1, F(h)=(\Phi U-g n) h+\Phi V h^{\prime}+g v H^{\prime}$, and $G(h)=\left(\Phi U^{\prime}-g n\right) h$ $+\Phi V g^{\prime}+g v G^{\prime}$. Since $h^{\prime} \equiv p H^{\prime}(\bmod g k)$ if $H H^{\prime} \equiv-1(\bmod g k)$ and since an integer $\delta$ exists such that $p \delta \equiv 1(\bmod g k)$ we see that $F(h) \equiv(\Phi U-g n) h$ $+(\Phi V+\delta g v) h^{\prime}(\bmod g k)$. Also, since $h^{\prime} \equiv 2 g^{\prime}(\bmod g k)$, and $h^{\prime} \equiv 2 p G^{\prime}(\bmod g k)$ if $2 H G^{\prime} \equiv-1(\bmod g k)$ and since integers $\sigma$ and $\omega$ exist such that $2 \sigma \equiv 1(\bmod g k)$ and $2 p \omega \equiv 1(\bmod g k)$ we have $G(h) \equiv\left(\Phi U^{\prime}-g n\right) h+\left(\sigma \Phi V^{\prime}+\omega g v\right) h^{\prime}(\bmod g k)$.

Following Lehner's procedure [2], these sums may now be written in the form $S_{i}=O\left(K_{i} \log k\right)$ where $K_{i}$ is a complete Kloosterman sum. The desired estimates are now obtained as in the proof of Theorem 2 in [1].

\section{A CONVERGENT SERIES FOR $q_{a}(n)$}

9. By Cauchy's integral formula and (2.2) we have

$$
q_{a}(n)=\frac{1}{2 \pi i} \int_{C} x^{-n-1} G_{a}(x) d x=\sum_{h, k}^{\prime} \frac{1}{2 \pi i} \int_{\xi_{h k}} x^{-n-1} G_{a}(x) d x
$$

where $0 \leqq h<k \leqq N$ and $\xi_{h k}$ are the Farey arcs of order $N$ of $C$, the circle $|x|=\exp \left\{-2 \pi N^{-2}\right\}$. If, on $\xi_{h k}$, we introduce the variable $\phi$ by setting $x=\exp \left\{-2 \pi N^{-2}+2 \pi i h / k+2 \pi i \phi\right\}$ and then write $w=N^{-2}-i \phi, z=w k$, we have

$$
q_{a}(n)=\sum_{h k}^{\prime} \exp \{-2 \pi i n h / k\} \int_{-\theta^{\prime}}^{\theta^{\prime \prime}} G_{a}(\exp \{2 \pi i h / k-2 \pi z / k\}) \exp \{2 \pi n w\} d \phi .
$$

Here $\theta^{\prime}=1 / k\left(k+k_{1}\right)$ and $\theta^{\prime \prime}=1 / k\left(k+k_{2}\right)$ where $h_{1} / k_{1}<h / k<h_{2} / k_{2}$ are consecutive terms in the Farey series of order $N$.

We now split the sum over $k$ into four parts $q_{a}(n, 1), q_{a}(n, 2), q_{a}(n, 3), q_{a}(n, 4)$ according as $k$ satisfies the requirements given in (3.23), (3.24), (3.25), (3.26) respectively. Selecting $\beta$ so that $a_{1} \beta \equiv 1(\bmod p)$ we have by $(3.23)$

$$
\begin{gathered}
q_{a}(n, 1)=\sum_{b_{1}=1}^{(p-1) / 2} \sum_{h, k}^{\prime} w_{a}(h, k) \exp \{-2 \pi i n h / k\} \int_{-\theta^{\prime}}^{\theta^{\prime \prime}} \sum_{v=0}^{\infty} q_{b}(v) \exp \left\{2 \pi i h^{\prime} v / k\right\} \\
\cdot \exp \left\{-\left(\pi / k^{2} w\right)(2 v+B / 6 p)+\pi w(2 n+A / 6 p)\right\} d \phi,
\end{gathered}
$$

where $h \equiv \pm b_{1} \beta(\bmod p)$.

If we split the sum over $v$ into two parts according as $2 v+B / 6 p$ is negative or nonnegative we find, using Rademacher's argument [3] and employing Theorem 2, that

$$
\begin{gathered}
q_{a}(n, 1)=2 \pi \sum_{k=1}^{N} \sum_{b_{1}=1}^{(p-1) / 2} \sum_{v<-B / 12 p} q_{b}(v) A_{k b}(n, v) L_{k b}(n, v) \\
+O\left(n^{1 / 3} N^{-1 / 3+\varepsilon} \exp \left\{2 \pi n N^{-2}\right\}\right)
\end{gathered}
$$


where $2 p \mid k$. Here,

$$
A_{k b}(n, v)=\sum_{h \bmod k}^{\prime} w_{a}(h, k) \exp \left\{-2 \pi i\left(n h-v h^{\prime}\right) / k\right\}
$$

where $h \equiv \pm b_{1} \beta(\bmod p) ;$ and

(9.3) $L_{k b}(n, v)=\left\{\begin{array}{l}k^{-1}\{(-B-12 v p) /(12 n p+A)\}^{1 / 2} \\ \cdot I_{1}\left\{\pi(12 n p+A)^{1 / 2}(-B-12 v p)^{1 / 2} / 3 p k\right\} \text { if } n>-A / 12 p, \\ -(B+12 v p) \pi / 6 p k^{2} \text { if } n=-A / 12 p,\end{array}\right.$

where $I_{1}(z)$ is the Bessel function.

As an example of a case for which $n=-A / 12 p$, if we take $p=71$, $a=\{22,23,25,26,27,28,29,30,31,32,33,34\}$, we find that $-A / 12 p=30$. The case $n<-A / 12 p$ is of no interest since the final formula for $q_{a}(n)$ requires the calculation of $q_{a}(v)$ for $v<-A / 12 p$.

From (3.24) we have

$$
\begin{aligned}
q_{a}(n, 2)= & \sum_{b_{1}=1}^{(p-1) / 2} \sum_{h, k}^{\prime} W_{a}(h, k) \exp \{-2 \pi i n h / k\} \int_{-\theta^{\prime}}^{\theta^{\prime \prime}} \sum_{v=0}^{\infty} s_{b}(v) \exp \left\{2 \pi i g^{\prime} v / k\right\} \\
& \cdot \exp \left\{-\left(\pi / k^{2} w\right)\left(v-B^{*} / 6 p\right)+\pi w(2 n+A / 6 p)\right\} d \phi,
\end{aligned}
$$

where $h \equiv \pm b_{1} \beta(\bmod p)$.

Splitting the sum over $v$ into two parts according as $v-B^{*} / 6 p$ is negative or nonnegative, utilizing Rademacher's method [3] and making use of Theorem 3 we have

$$
\begin{aligned}
q_{a}(n, 2)= & 2 \pi \sum_{k=1}^{N} \sum_{b_{1}=1}^{(p-1) / 2} \sum_{v<B^{*} / 6 p} s_{b}(v) B_{k b}(n, v) M_{k b}(n, v) \\
& +O\left(n^{1 / 3} N^{-1 / 3+\varepsilon} \exp \left\{2 \pi n N^{-2}\right\}\right)
\end{aligned}
$$

where $p \mid k, 2 \times k$.

$$
B_{k b}(n, v)=\sum_{h \bmod k}^{\prime} W_{a}(h, k) \exp \left\{-2 \pi i\left(n h-v g^{\prime}\right) / k\right\}
$$

where $h \equiv \pm b_{1} \beta(\bmod p)$, and

$$
M_{k b}(n, v)=\left\{\begin{array}{c}
k^{-1}\left\{\left(B^{*}-6 v p\right) /(12 n p+A)\right\}^{1 / 2} \\
\cdot I_{1}\left\{\pi(12 n p+A)^{1 / 2}\left(B^{*}-6 v p\right)^{1 / 2} / 3 p k\right\} \text { if } n>-A / 12 p, \\
\left(B^{*}-6 v p\right) \pi / 6 p k^{2} \text { if } n=-A / 12 p .
\end{array}\right.
$$

From (3.25) we have

$$
\begin{aligned}
& \left.q_{a}(n, 3)=2^{r} \sum_{h, k}^{\prime}(-1)^{\mu}\left(\prod_{j=1}^{r} \cos \pi \alpha_{j} / p\right)\right) z_{a}(h, k) \exp \{-2 \pi i n h / k\} \\
& \cdot \int_{-\theta^{\prime}}^{\theta^{\prime \prime}} \sum_{v=0}^{\infty} d_{a k}(v) \exp \left\{2 \pi i H^{\prime} v / k\right\} \exp \{-(\pi / K k w)(2 v+r / 6)+\pi w(2 n+A / 6 p)\} d \phi .
\end{aligned}
$$


Since $2 v+r / 6>0$ we have by the argument detailed in [3], and employing Theorem 4

$$
q_{a}(n, 3)=O\left(n^{1 / 3} N^{-1 / 3+\varepsilon} \exp \left\{2 \pi n N^{-2}\right\}\right) .
$$

From (3.26) we have

$$
\begin{aligned}
q_{a}(n, 4)= & \sum_{h, k}^{\prime} Z_{a}(h, k) \exp \{-2 \pi i n h / k\} \int_{-\theta^{\prime}}^{\theta^{\prime \prime}} \sum_{v=0}^{\infty} e_{a k}(v) \exp \left\{2 \pi i G^{\prime} v / k\right\} \\
& \cdot \exp \{-(\pi / K k w)(v-r / 12)+\pi w(2 n+A / 6 p)\} d \phi .
\end{aligned}
$$

If we split the sum over $v$ into two parts according as $v-r / 12$ is negative or nonnegative we have, using Theorem 5 ,

$$
\begin{aligned}
q_{a}(n, 4)= & 2 \pi \sum_{k=1}^{N} \sum_{v<r / 12} e_{a k}(v) C_{k}(n, v) N_{k}(n, v) \\
& +O\left(n^{1 / 3} N^{-1 / 3+\varepsilon} \exp \left\{2 \pi n N^{-2}\right\}\right) . \\
C_{k}(n, v)= & \sum_{h \bmod k} Z_{a}(h, k) \exp \left\{-2 \pi i\left(n h-v G^{\prime}\right) / k\right\} . \\
N_{k}(n, v)= & \left\{\begin{array}{l}
k^{-1}\{(r / 2-6 v) /(12 n p+A)\}^{1 / 2} \\
\cdot I_{1}\left\{\pi(12 n p+A)^{1 / 2}(r / 2-6 v)^{1 / 2} / 3 p k\right\} \text { if } n>-A / 12 p, \\
(r / 2-6 v) \pi / 6 p k^{2} \text { if } n=A / 12 p .
\end{array}\right.
\end{aligned}
$$

Letting $N \rightarrow \infty$ in (9.1), (9.4), (9.7), (9.8) we have

THEOREM 6. The number, $q_{a}(n)$, of partitions of a positive integer $n$, $n \geqq-A / 12 p$, into distinct positive summands of the form $p m \pm a_{j}, a_{j} \in a$, is given by the convergent series

$$
\begin{aligned}
q_{a}(n)= & 2 \pi \sum_{k=1}^{\infty} \sum_{b_{1}=1}^{(p-1) / 2} \sum_{v<-B / 12 p} q_{b}(v) A_{k b}(n, v) L_{k b}(n, v) \\
& +2 \pi \sum_{k=1}^{\infty} \sum_{b_{1}=1}^{(p-1) / 2} \sum_{v<B^{*} / 6 p} s_{b}(v) B_{k b}(n, v) M_{k L}(n, v) \\
& +2 \pi \sum_{k=1}^{\infty} \sum_{v<r / 12} e_{a k}(v) C_{k}(n, v) N_{k}(n, v)
\end{aligned}
$$

where $A_{k b}, L_{k b}, B_{k b}, M_{k b}, C_{k}, N_{k}$ are given by (9.2), (9.3), (9.5), (9.6),(9.9), (9.10) respectively. In the first sum $2 p \mid k$; in the second $p \mid k, 2 \times k$; in the third $p \nmid k$, $2 \nmid k$.

\section{AsYmptotic Formulas}

10. As in the case of $p_{a}(n)$ the dominant term in the series representing $q_{a}(n)$ is that for which $k=1$. Letting $G(v)=(r / 2-6 v)^{1 / 2}, T=\pi(12 n p+A)^{1 / 2} / 3 p$, $W=\Sigma_{v<r / 12} e_{a 1}(v) G(v) I_{1}\{T G(v)\}$ we have 
THEOREM 7. As $n \rightarrow \infty$

$$
q_{a}(n)=2 \pi W(12 n p+A)^{-1 / 2}\left(1+O\left(\exp \left\{-c n^{2 / 2}\right\}\right)\right)
$$

where $c>0$.

The proof is essentially the same as that of Theorem 7 in [1] and is therefore omitted. We remark only that one must establish that if $-B>0\left(B^{*}>0\right)$ and $M$ is the maximum possible value of $-B\left(B^{*}\right)$ then $k^{-1} M^{1 / 2}-(r / 2)^{1 / 2}<0$ if $k \geqq p$. This is easily done as follows.

Since $B$ is a decreasing function of each $b_{j}$ the maximal value of $-B$ is $M=-r p^{2}+6 p \sum j-6 \sum j^{2}$ where $(p+1) / 2-r \leqq j \leqq(p-1) / 2$. An easy calculation yields $M=r p^{2} / 2+r\left(1-4 r^{2}\right) / 2<r p^{2} / 2$. (The maximal value of $B^{*}$ is $M=r p^{2} / 2-6 \sum_{j=1}^{r} j^{2}<r p^{2} / 2$.) Since $k \geqq p$ we have $k^{-2} M \leqq p^{-2} M<r / 2$.

Since $W \sim(r / 2)^{1 / 2} I_{1}\left\{T(r / 2)^{1 / 2}\right\}$ (see (11.13) in [1]) we have from Theorem 7

Corollary 7.1. As $n \rightarrow \infty$

$$
q_{a}(n)=\pi(2 r /(12 n p+A))^{1 / 2} I_{1}\left\{T(r / 2)^{1 / 2}\right\}\left(1+O\left(\exp \left\{-c n^{1 / 2}\right\}\right)\right) .
$$

Finally, since $I_{1}(z)=e^{z}(2 \pi z)^{-1 / 2}\left(1+O\left(z^{-1}\right)\right)$ as $z \rightarrow \infty$, we have

Corollary 7.2. As $n \rightarrow \infty$

$$
q_{a}(n)=(6 p)^{1 / 2}(r / 2)^{1 / 4}(12 n p+A)^{-3 / 4} \exp \left\{T(r / 2)^{1 / 2}\right\}\left(1+O\left(n^{-1 / 2}\right)\right) .
$$

\section{REFERENCES}

1. P. Hagis, Jr., A problem on partitions with a prime modulus $p \geqq 3$, Trans. Amer. Math. Soc. 102 (1962), 30-62.

2. J. Lehner, A partition function connected with the modulus five, Duke Math. J. 8 (1941), 631-655.

3. H. Rademacher, The Fourier coefficients of the modular invariant $J(\tau)$, Amer. J. Math. 60 (1938), 501-512.

TEMPLE UNIVERSITY,

Philadelphia, Pennsylvania 